\title{
A Coupled BEM Model for the Dynamic Analysis of a Pile Embedded in a Half-space Soil Covered by a Water Layer
}

\author{
Xu Zhang and Jian-Fei Lu*
}

Department of Civil Engineering, Jiangsu University, Zhenjiang, Jiangsu, 212013, P.R. China

\begin{abstract}
Dynamic analysis of a pile embedded in a half-space soil covered by a water layer is crucial for the designs of the pile foundations for bridges, docks and offshore platforms etc. In this paper, a coupled boundary element method (BEM) model is developed to evaluate the dynamic response of the pile. In the proposed model, the pile and half-space soil are treated as elastic media, while the water layer is considered as an acoustic medium. Three BEM formulations are established for the pile, half-space soil and water layer by means of the boundary element method (BEM), respectively. Using the three BEM formulations as well as the continuity conditions at the interfaces between three regions, a coupled BEM model for the pile-soil-water system is established. To validate the proposed model, results due to our model are compared with existing results. With the coupled BEM model for the pile-soil-water system, dynamic response of the pile is investigated. Presented numerical results show that when the pile is subjected to an axial load and torque, resonance phenomena is not obvious. However, when the pile is subjected to a horizontal load and moment, resonance phenomena is pronounced and the pile-soil modulus and density ratios have a considerable influences on resonant frequencies.
\end{abstract}

Keywords: Pile; the half-space soil; the water layer; the boundary element method (BEM); compliance.

\section{INTRODUCTION}

The analysis of the dynamic responses of pile foundations embedded in the half-space soil has received considerable attentions during the past few decades. Makris $[1,2]$ used the Winkler model to investigate the dynamic interaction between piles and soil. Kuhlemeyer $[3,4]$ and $\mathrm{Wu} \&$ Finn [5] employed the finite element method to study the dynamic response of piles. Zeng \& Rajapakse $[6,7]$ as well as Rajapakse \& Shah $[8,9]$ utilized the integral equation method to investigate the dynamic interaction between piles and the half-space soil. As the integral equation method can simplify piles as one-dimensional structures and handle the half-space soil by semi-analytical methods, the threedimensional pile-soil interaction problem can thus be simplified substantially by the method. As a result, the integral equation method has been employed to solve various pilesoil interaction problems so far [10-13]. Besides the aforementioned methods, the boundary element method has also been used widely to deal with the pile-soil interaction problem. For example, Mamoon [14] used a hybrid boundary element formulation to evaluate the impedance and compliance functions of piles and pile groups. Sen [15] used a boundary element formulation for the dynamic analysis of axially and laterally loaded piles and pile groups embedded in a hysteretic elastic half-space. Wang \& Banerjee [16] employed an axisymmetric boundary element formulation for the analysis of pile-soil interaction. Moreover, the steady

*Address correspondence to this author at the Department of Civil Engineering, Jiangsu University, Zhenjiang, Jiangsu, 212013, P.R. China;

Tel: 0086-15951284152; Fax: 0086-88780030;

E-mail: ljfdoctor@yahoo.com state dynamic response of a pile group embedded in a poroelastic half-space soil is investigated by means of the boundary element method for a saturated porous medium in [17].

It is noted that although many researches concerning dynamic pile-soil interaction problems have been conducted so far, most existing researches are restricted to the dynamic analysis of piles embedded in a half-space soil. However, for the pile foundations associated with cross-sea bridges, docks and offshore platforms, the half-space soil is usually covered by a water layer. In this case, the influence of the water layer on the dynamic response of pile foundations should be accounted for. As a result, to estimate the dynamic response of piles in this situation properly, the coupling between the pile, half-space soil and water layer should be taken into account. It is worth emphasizing that dynamic characteristics of this kind of piles are crucial for the dynamic analysis of cross-sea bridges, docks and offshore platforms subjected to various dynamic loads, such as, seismic waves, current loads, water waves, wind loads and moving loads due to vehicles etc. However, as noted above, there have been very few researches carried out about the dynamic responses of piles embedded in a half-space soil covered by a water layer. As a result, to accomplish a successful dynamic analysis for the pile foundations of bridges and various offshore facilities, it is of significance to develop a coupled model for the dynamic analysis of piles embedded in a half-space soil with an overlying water layer.

The objective of this study is to develop a coupled pilesoil-water model to analyze the dynamic response of a single pile embedded in a half-space soil covered by a water layer. 
In the proposed model, the pile and half-space soil are treated as elastic media, while the water layer is considered as an acoustic medium. Three boundary element method (BEM) formulations are established for the pile, half-space soil and water layer by means of the boundary element method, respectively. Using the three BEM formulations as well as the continuity conditions at the interfaces between three regions, a coupled BEM model for the pile-soil-water system is established. With the proposed BEM model for the pile-soil-water system, some numerical results for the dynamic response of the pile are presented in this study.

\section{BOUNDARY INTEGRAL EQUATIONS FOR THE PILE, HALF-SPACE SOIL AND WATER LAYER}

As noted above, the pile and half-space soil in this study are treated as the elastic medium, while the water layer is considered as an acoustic medium. In this section, the boundary integral equations for the elastic medium and acoustic medium will be outlined.

\subsection{Boundary Integral Equation for the Elastic Medium}

As this paper is concerned with the frequency domain analysis of a pile embedded in a half-space soil overlain by a water layer, the Fourier transform for the time is involved. In this study, the Fourier transform for the time is defined as follows [18]:

$$
\hat{f}(\omega)=\int_{-\infty}^{+\infty} f(t) e^{-\mathrm{i} \omega t} d t, f(t)=\frac{1}{2 \pi} \int_{-\infty}^{+\infty} \hat{f}(\omega) e^{\mathrm{i} \omega t} d \omega
$$

in which $t$ and $\omega$ represent the time and angular frequency, respectivley, the variable with a caret denotes the frequency domain variable. Note that as this study is restricted to the frequency domain analysis of the pile, for brevity, the caret denoting the frequency domain variable will be dropped for all forthcoming frequency domain variables.

The equation of motion for an elastic medium in the frequency domain is as follows [19]:

$\mu u_{i, j j}+(\lambda+\mu) u_{j, j i}=-\rho \omega^{2} u_{i}$

where $\lambda$ and $\mu$ are the Lame constants of the elastic medium; $u_{i}$ is the displacement; $\rho$ is the density. The constitutive relation for the elastic medium is as follows [19]:

$\sigma_{i j}=2 \mu \varepsilon_{i j}+\lambda \delta_{i j} e$

where $\sigma_{i j}$ denotes the stress component of the elastic medium; $\varepsilon_{i j}$ is the strain component; $e$ denotes the bulk strain; $\delta_{i j}$ denotes the Kronecker delta.

Based on the dynamic reciprocal theorem, the frequency domain boundary integral equation for an elastic medium can be obtained as follows [20]:

$$
c_{i j} u_{j}(\xi)=\int_{\Gamma}\left[U_{i j}^{(G)}(\mathbf{x}, \mathbf{y}) t_{j}(\psi)-T_{i j}^{(G)}(\xi, \psi) u_{j}(\psi)\right] d \Gamma(\psi)
$$

where $U_{i j}^{(G)}, T_{i j}^{(G)}$ are the Green's functions for the elastic medium and are given in APPENDIX [21]; $u_{j}, t_{j}$ are the displacements and tractions along the boundary of the elastic medium; $c_{i j}$ is the coefficients for the boundary and $\Gamma$ denotes the boundary of the elastic medium.

\subsection{Boundary Integral Equation for An acoustic Medium}

As noted previously, the water layer in this study is treated as an acoustic medium. Thus, for the water layer, its pressure is described by the following Helmholtz equation [22]:

$$
\nabla^{2} p+k_{w}^{2} p=0, k_{w}=\frac{\omega}{v_{w}}
$$

in which $p$ is the pressure of the water layer; $k_{w}$ is the wavenumber of the water; $v_{w}$ is the acoustic velocity of water. The displacement of the water layer has the form [22]:

$\mathbf{q}=\frac{1}{\rho_{w} \omega^{2}} \nabla p$

where $\mathbf{q}$ denotes the displacement vector for the water layer and $\rho_{w}$ is the density of water.

Using the dynamic reciprocal theorem, the frequency domain boundary integral equation for the water layer is as follows [22, 23]:

$$
c p(\mathbf{x})=\int_{\Gamma}\left[p^{(G)}(\mathbf{x}, \mathbf{y}) q_{n}(\mathbf{y})-q_{n}^{(G)}(\mathbf{x}, \mathbf{y}) p(\mathbf{y})\right] d \Gamma(\mathbf{y})
$$

where $p^{(G)}$ and $q_{n}^{(G)}$ are the Green's functions for the water; $c$ is the coefficient for the boundary and depends upon the local geometry at the point $\mathbf{x}$. Note that $q_{n}(\mathbf{y})$ is the displacement of the water along the normal direction and given by

$q_{n}(\mathbf{y})=\frac{1}{\rho_{w} \omega^{2}} \frac{\partial p(\mathbf{y})}{\partial \mathbf{n}(\mathbf{y})}$

where $\mathbf{n}(\mathbf{y})$ is the outward normal for the boundary point $\mathbf{y}$ at $\Gamma$. The Green's functions in equation (7) are given by

$$
p^{(G)}(\mathbf{x}, \mathbf{y})=\frac{\rho_{w} \omega^{2}}{4 \pi}\left[\frac{1}{r} e^{-i k_{w} r}\right], \quad q_{n}^{(G)}(\mathbf{x}, \mathbf{y})=\frac{1}{4 \pi} \frac{\partial}{\partial \mathbf{n}(\mathbf{y})}\left[\frac{1}{r} e^{-i k_{w} r}\right]
$$

in which $r=\sqrt{\left(y_{j}-x_{j}\right)\left(y_{j}-x_{j}\right)}$.

\section{BOUNDARY ELEMENT FORMULATIONS FOR THE PILE, HALF-SPACE SOIL AND WATER LAYER}

In this section, according to the boundary integral equations for the elastic medium and acoustic medium, three boundary element formulations for the pile, half-space soil and water layer are established, respectively. As shown in Fig. (1), for a pile embedded in a half-space soil overlain by a water layer, three interfaces and two boundaries exist, that is, the interface between the pile and half-space soil $\left(\Gamma_{1}\right)$, the interface between the pile and water layer $\left(\Gamma_{2}\right)$, the boundary of the pile top $\left(\Gamma_{3}\right)$, the interface between the half-space 


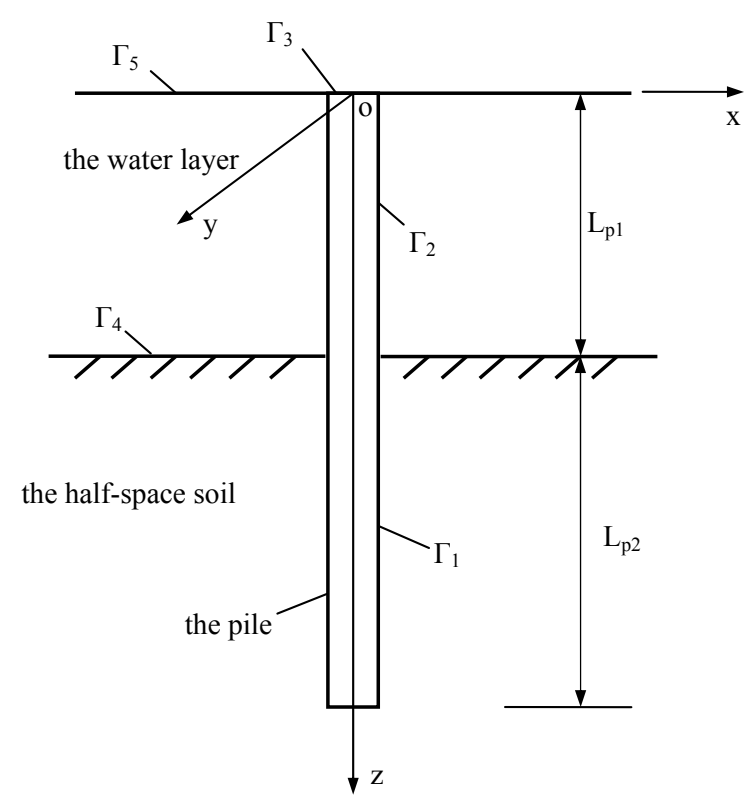

Fig. (1). A schematic illustration of a pile embedded in a half-space soil covered by a water layer.

soil and water layer $\left(\Gamma_{4}\right)$ and the boundary of the water layer $\left(\Gamma_{5}\right)$. Thus, the overall boundary of the pile consists of $\Gamma_{1}, \Gamma_{2}$ and $\Gamma_{3}$, respectively; the boundary of the half-space soil is composed of $\Gamma_{1}$ and $\Gamma_{4}$, respectively; the boundary of the water layer comprises $\Gamma_{2}, \Gamma_{4}$ and $\Gamma_{5}$, respectively.

The frequency domain integral equations for the pile, half-space soil and water layer can be discretized if suitable numbers of boundary elements are used to cover the corresponding boundaries. In this study, for the convenience of enforcing the continuity conditions between different regions, all boundaries of the pile, half-space soil and water layer are discretized by the same type of iso-parametric boundary elements.

Suppose that the boundaries of the pile, half-space soil and water layer are discretized by the same type of isoparametric elements with each boundary element containing $N_{n d}$ nodes. Thus, for a point $x$ inside the j-th element, the following interpolation formulae hold for the pile, half-space soil and water layer [24]

$$
\begin{aligned}
& x_{\alpha}^{(j)}(\xi, \eta)=\sum_{n=1}^{N_{n d}} N_{n}(\xi, \eta) x_{n \alpha}^{(j)}, \quad u_{\alpha}^{(j)}\left(x_{\beta}^{(j)}\right)=\sum_{n=1}^{N_{n d}} N_{n}(\xi, \eta) u_{n \alpha}^{(j)}, \\
& t_{\alpha}^{(j)}\left(x_{\beta}^{(j)}\right)=\sum_{n=1}^{N_{n d}} N_{n}(\xi, \eta) t_{n \alpha}^{(j)}, \\
& p^{(j)}\left(x_{\beta}^{(j)}\right)=\sum_{n=1}^{N_{n d}} N_{n}(\xi, \eta) p_{n}^{(j)}, \quad q_{n}^{(j)}\left(x_{\beta}^{(j)}\right)=\sum_{n=1}^{N_{n d}} N_{n}(\xi, \eta) q_{n n}^{(j)}, \\
& \alpha, \beta=1 \sim 3
\end{aligned}
$$

where the superscript $\mathrm{j}$ denotes the element number; $n$ is the local node number; $\xi, \eta$ are the intrinsic coordinates corre- sponding to the point $x ; N_{n}(\xi, \eta)$ is the n-th shape function; $x_{\alpha}^{(j)}$ and $x_{n \alpha}^{(j)}$ are the coordinates for the point $x$ and $\mathrm{n}$-th node of the $\mathrm{j}$-th element in the global coordinate system; $u_{\alpha}^{(j)}$ and $u_{n \alpha}^{(j)}$ denote the displacements at the point $x$ and n-th node of the j-th element; $t_{\alpha}^{(j)}, t_{n \alpha}^{(j)}, p^{(j)}, p_{n}^{(j)}, q_{n}^{(j)}$ and $q_{n n}^{(j)}$ have the similar meanings.

\subsection{Boundary Element Formulation for the Pile}

By using the boundary integral equation for the pile, using the discretization scheme as outlined in equation (10) and performing the integration of the shape function kernel products over all the boundary elements for the pile, the following boundary element formulation for the pile is obtained:

$$
\mathbf{H}^{(p)} \mathbf{U}^{(p)}=\mathbf{G}^{(p)} \mathbf{T}^{(p)}
$$

where the superscript $p$ denotes the pile; $\mathbf{G}^{(p)}$ and $\mathbf{H}^{(p)}$ in equation (11) are the coefficient matrices obtained by integrating shape function kernel products over all boundary elements of the pile; $\mathbf{U}^{(p)}$ and $\mathbf{T}^{(p)}$ are the generalized displacement and traction vectors of the nodes of the boundary elements of the pile.

Dividing $\mathbf{U}^{(p)}$ and $\mathbf{T}^{(p)}$ into three parts corresponding to $\Gamma_{1}, \Gamma_{2}$ and $\Gamma_{3}$, respectively, and partitioning the coefficient matrices $\mathbf{G}^{(p)}$ and $\mathbf{H}^{(p)}$, accordingly, one has the following matrix equation:

$$
\left[\begin{array}{lll}
\mathbf{H}_{1}^{(p)} & \mathbf{H}_{2}^{(p)} & \mathbf{H}_{3}^{(p)}
\end{array}\right]\left\{\begin{array}{l}
\mathbf{U}_{1}^{(p)} \\
\mathbf{U}_{2}^{(p)} \\
\mathbf{U}_{3}^{(p)}
\end{array}\right\}=\left[\begin{array}{ll}
\mathbf{G}_{1}^{(p)} \mathbf{G}_{2}^{(p)} \mathbf{G}_{3}^{(p)}
\end{array}\right]\left\{\begin{array}{c}
\mathbf{T}_{1}^{(p)} \\
\mathbf{T}_{2}^{(p)} \\
\mathbf{T}_{3}^{(p)}
\end{array}\right\}
$$

where the subscripts 1,2 and 3 represent the boundaries $\Gamma_{1}, \Gamma_{2}$ and $\Gamma_{3}$ of the pile, respectively (Fig. 1); $\mathbf{U}_{j}^{(p)}$ and $\mathbf{T}_{j}^{(p)}(\mathrm{j}=1 \sim 3)$ denote the generalized displacement and traction vectors for the boundaries $\Gamma_{j}(\mathrm{j}=1 \sim 3)$ of the pile, respectively; $\mathbf{H}_{1}^{(p)}, \mathbf{H}_{2}^{(p)}, \mathbf{H}_{3}^{(p)}, \mathbf{G}_{1}^{(p)}, \mathbf{G}_{2}^{(p)}$ and $\mathbf{G}_{3}^{(p)}$ are the sub-matrices of the coefficient matrices $\mathbf{G}^{(p)}$ and $\mathbf{H}^{(p)}$ of the pile, corresponding to the boundaries $\Gamma_{1}, \Gamma_{2}$ and $\Gamma_{3}$, respectively.

In the conventional BEM formulation, the generalized displacement and traction vectors $\mathbf{U}_{j}^{(p)}$ and $\mathbf{T}_{j}^{(p)}(\mathrm{j}=1 \sim 3)$ for the boundaries $\Gamma_{j}(\mathrm{j}=1 \sim 3)$ of the pile have the following expressions:

$$
\begin{aligned}
& \mathbf{U}_{j}^{(p)}=\left[\mathbf{u}^{(p) T}\left(\mathbf{x}_{1}^{(j)}\right), \mathbf{u}^{(p) T}\left(\mathbf{x}_{2}^{(j)}\right), \cdots, \mathbf{u}^{(p) T}\left(\mathbf{x}_{N_{j}}^{(j)}\right)\right]^{T}, \mathbf{j}=1 \sim 3, \\
& \left.\mathbf{T}_{j}^{(p)}=\left[\mathbf{t}^{(p) T}\left(\mathbf{x}_{1}^{(j)}\right), \mathbf{t}^{(p) T}\left(\mathbf{x}_{2}^{(j)}\right)\right), \cdots, \mathbf{t}^{(p) T}\left(\mathbf{x}_{N_{j}}^{(j)}\right)\right]^{T}, \mathrm{j}=1 \sim 3, \\
& \mathbf{u}^{(p)}\left(\mathbf{x}_{i}^{(j)}\right)=\left[u_{x}^{(p)}\left(\mathbf{x}_{i}^{(j)}\right), u_{y}^{(p)}\left(\mathbf{x}_{i}^{(j)}\right), u_{z}^{(p)}\left(\mathbf{x}_{i}^{(j)}\right)\right]^{T}, \quad i=1 \sim N_{j}, \\
& \mathrm{j}=1 \sim 3,
\end{aligned}
$$




$$
\begin{aligned}
& \mathbf{t}^{(p)}\left(\mathbf{x}_{i}^{(j)}\right)=\left[t_{x}^{(p)}\left(\mathbf{x}_{i}^{(j)}\right), t_{y}^{(p)}\left(\mathbf{x}_{i}^{(j)}\right), t_{z}^{(p)}\left(\mathbf{x}_{i}^{(j)}\right)\right]^{T}, \quad i=1 \sim N_{j}, \\
& \mathrm{j}=1 \sim 3
\end{aligned}
$$

in which $\mathbf{x}_{i}^{(j)}\left(\mathrm{j}=1 \sim 3, i=1 \sim N_{j}\right)$ denotes the i-th node of the boundary $\Gamma_{j}(\mathrm{j}=1 \sim 3)$ of the pile; $\mathbf{u}^{(p)}\left(\mathbf{x}_{i}^{(j)}\right)$ and $\mathbf{t}^{(p)}\left(\mathbf{x}_{i}^{(j)}\right)$ are the displacement and traction vectors for the ith node at the boundary $\Gamma_{j}(\mathrm{j}=1 \sim 3)$ of the pile; $N_{j}$ is the number of the nodes belonging to the boundary $\Gamma_{j}$ $(\mathrm{j}=1 \sim 3)$ of the pile and the superscript $T$ denotes the transpose of a vector or a matrix. To facilitate the implementation of the continuity conditions between the pile and water layer, it is necessary to rearrange the displacement and traction vectors $\mathbf{U}_{2}^{(p)}$ and $\mathbf{T}_{2}^{(p)}$ along the boundary $\Gamma_{2}$ as follows:

$$
\begin{aligned}
& \mathbf{U}_{2}^{(p)}=\left[\mathbf{U}_{2 x}^{(p) T}, \mathbf{U}_{2 y}^{(p) T}, \mathbf{U}_{2 z}^{(p) T}\right]^{T}, \mathbf{T}_{2}^{(p)}=\left[\mathbf{T}_{2 x}^{(p) T}, \mathbf{T}_{2 y}^{(p) T}, \mathbf{T}_{2 z}^{(p) T}\right]^{T}, \\
& \mathbf{U}_{2 \alpha}^{(p) T}=\left[u_{\alpha}^{(p)}\left(\mathbf{x}_{1}^{(2)}\right), u_{\alpha}^{(p)}\left(\mathbf{x}_{2}^{(2)}\right), \cdots, u_{\alpha}^{(p)}\left(\mathbf{x}_{N_{2}}^{(2)}\right)\right]^{T}, \\
& \mathbf{T}_{2 \alpha}^{(p)}=\left[t_{\alpha}^{(p)}\left(\mathbf{x}_{1}^{(2)}\right), t_{\alpha}^{(p)}\left(\mathbf{x}_{2}^{(2)}\right), \cdots, t_{\alpha}^{(p)}\left(\mathbf{x}_{N_{2}}^{(2)}\right)\right]^{T}, \alpha=x, y, z, \\
& \mathbf{x}_{i}^{(2)} \in \Gamma_{2}, i=1 \sim N_{2}
\end{aligned}
$$

\subsection{Boundary Element Formulation for the Half-space Soil}

Analogously, the boundary element formulation for the half-space soil can also be established by discretizing the corresponding boundary integral equation and integrating the shape function kernel products over all boundary elements of the half-space soil. Dividing boundary displacement and traction vectors into two parts corresponding to $\Gamma_{1}$ and $\Gamma_{4}$, respectively, and partitioning the coefficient matrices accordingly, one has the following boundary element formulation for the half-space soil:

$$
\left[\begin{array}{ll}
\mathbf{H}_{1}^{(s)} & \mathbf{H}_{4}^{(s)}
\end{array}\right]\left\{\begin{array}{c}
\mathbf{U}_{1}^{(s)} \\
\mathbf{U}_{4}^{(s)}
\end{array}\right\}=\left[\begin{array}{ll}
\mathbf{G}_{1}^{(s)} & \mathbf{G}_{4}^{(s)}
\end{array}\right]\left\{\begin{array}{c}
\mathbf{T}_{1}^{(s)} \\
\mathbf{T}_{4}^{(s)}
\end{array}\right\}
$$

where the superscript $s$ denotes the half-space soil; $\mathbf{U}_{j}^{(s)}$ and $\mathbf{T}_{j}^{(s)}(\mathrm{j}=1,4)$ denote the generalized displacement and traction vectors for the boundaries $\Gamma_{j}(j=1,4)$ of the halfspace soil, respectively and they have the similar representations as shown in equation (13); $\mathbf{H}_{1}^{(s)}, \mathbf{H}_{4}^{(s)}, \mathbf{G}_{1}^{(s)}$ and $\mathbf{G}_{4}^{(s)}$ are the sub-matrices of the coefficient matrices of the half-space soil, corresponding to the boundaries $\Gamma_{1}$ and $\Gamma_{4}$ of the halfspace soil, respectively. Similarly, for convenience of enforcing the continuity conditions between the half-space soil and water layer, the generalized displacement and traction vectors at the boundary $\Gamma_{4}$ of the half-space soil are rearranged as follows:

$\mathbf{U}_{4}^{(s)}=\left[\mathbf{U}_{4 x}^{(s) T}, \mathbf{U}_{4 y}^{(s) T}, \mathbf{U}_{4 z}^{(s) T}\right]^{T}, \mathbf{T}_{4}^{(s)}=\left[\mathbf{T}_{4 x}^{(s) T}, \mathbf{T}_{4 y}^{(s) T}, \mathbf{T}_{4 z}^{(s) T}\right]^{T}$

where the generalized displacement and traction vectors $\mathbf{U}_{4 \alpha}^{(s)}$ and $\mathbf{T}_{4 \alpha}^{(s)}(\alpha=x, y, z)$ are given by the similar expressions as shown in equation (14).

\subsection{Boundary Element Formulation for the Water Layer}

By using the boundary integral equation for the water layer and implementing the similar boundary discretization scheme as with the pile and half-space soil, the boundary element formulation can also be obtained for the water layer. Likewise, dividing boundary unknowns into three parts corresponding to $\Gamma_{2}, \Gamma_{4}$ and $\Gamma_{5}$, respectively, and partitioning the coefficient matrices accordingly, one has the following boundary element formulation for the water layer:

$$
\begin{aligned}
& {\left[\mathbf{H}_{2}^{(w)} \mathbf{H}_{4}^{(w)} \mathbf{H}_{5}^{(w)}\right]\left\{\begin{array}{l}
\mathbf{P}_{2} \\
\mathbf{P}_{4} \\
\mathbf{P}_{5}
\end{array}\right\}=\left[\mathbf{G}_{2}^{(w)} \mathbf{G}_{4}^{(w)} \mathbf{G}_{5}^{(w)}\right]\left\{\begin{array}{l}
\mathbf{Q}_{n 2} \\
\mathbf{Q}_{n 4} \\
\mathbf{Q}_{n 5}
\end{array}\right\},} \\
& \mathbf{P}_{j}=\left[p\left(\mathbf{x}_{1}^{(j)}\right), p\left(\mathbf{x}_{2}^{(j)}\right), \cdots, p\left(\mathbf{x}_{N_{j}}^{(j)}\right)\right]^{T}, \\
& \mathbf{Q}_{n j}=\left[q_{n}\left(\mathbf{x}_{1}^{(j)}\right), q_{n}\left(\mathbf{x}_{2}^{(j)}\right), \cdots, q_{n}\left(\mathbf{x}_{N_{j}}^{(j)}\right)\right]^{T}, \\
& \mathbf{x}_{i}^{(j)} \in \Gamma_{j}, i=1 \sim N_{j}, \mathrm{j}=2,4,5
\end{aligned}
$$

where the superscript $w$ denotes the water layer; $\mathbf{P}_{j}$ and $\mathbf{Q}_{n j}(\mathrm{j}=2,4,5)$ denote the generalized pressure and normal displacement vectors along the boundaries $\Gamma_{j}(j=2,4,5)$ of the water layer, respectively; $\mathbf{H}_{2}^{(w)}, \mathbf{H}_{4}^{(w)}, \mathbf{H}_{5}^{(w)}, \mathbf{G}_{2}^{(w)}, \mathbf{G}_{4}^{(w)}$ and $\mathbf{G}_{5}^{(w)}$ are the sub-matrices of the coefficient matrices of the water layer, corresponding to the boundaries $\Gamma_{j}(j=2,4,5)$ of the water layer, respectively.

\section{THE COUPLED BEM MODEL FOR THE PILE, HALF-SPACE SOIL AND WATER LAYER}

By means of the boundary integral equations for the elastic medium and acoustic medium, the three boundary element formulations have been established for the pile, halfspace soil and water layer, respectively. In this section, the coupled pile-soil-water BEM model will be developed using the aforementioned three boundary element formulations as well as the boundary conditions and continuity conditions at the interfaces between the three regions.

\subsection{Boundary Conditions and Continuity Conditions at the Interfaces between the Three Regions}

Boundary conditions are given at the top of the pile $\left(\Gamma_{3}\right)$ and the surface of the water layer $\left(\Gamma_{5}\right)$. The stress boundary condition at the top of the pile $\left(\Gamma_{3}\right)$ is determined by the loads acting on the top of the pile. Thus, it is assumed that $\mathbf{T}_{3}^{(p)}$ along the boundary $\Gamma_{3}$ of the pile in equation (12) is known a priori. Also, as the surface of the water layer is supposed to be free of pressure and traction, the pressure vector $\mathbf{P}_{5}$ along the boundary $\Gamma_{5}$ of the water layer in equation (17) thus vanishes. 
At the interface between the pile and half-space soil $\left(\Gamma_{1}\right)$, the continuity conditions for the displacements and tractions have the following form (Fig. 1):

$$
\mathbf{U}_{1}^{(p)}=\mathbf{U}_{1}^{(s)}, \mathbf{T}_{1}^{(p)}=-\mathbf{T}_{1}^{(s)}
$$

At the interface between the pile and water layer $\left(\Gamma_{2}\right)$, the continuity conditions for displacements and tractions have the following form (Fig. 1):

$$
\begin{aligned}
& -\left(\mathbf{N}_{2 x} \mathbf{U}_{2 x}^{(p)}+\mathbf{N}_{2 y} \mathbf{U}_{2 y}^{(p)}\right)=\mathbf{Q}_{n 2}, \quad \mathbf{T}_{2 x}^{(p)}=-\mathbf{N}_{2 x} \mathbf{P}_{2}, \quad \mathbf{T}_{2 y}^{(p)}=-\mathbf{N}_{2 y} \mathbf{P}_{2}, \\
& \mathbf{T}_{2 z}^{(p)}=\mathbf{0}
\end{aligned}
$$

where $\mathbf{N}_{2 x}$ and $\mathbf{N}_{2 y}$ are the direction cosine matrices along the $x$ and $y$ directions for the nodes at the boundary $\Gamma_{2}$ of the pile, which are given by the following expressions:

$$
\mathbf{N}_{2 x}=\left[\begin{array}{ccc}
n_{x}\left(\mathbf{x}_{1}^{(2)}\right) & & 0 \\
& \ddots & \\
0 & & n_{x}\left(\mathbf{x}_{N_{2}}^{(2)}\right)
\end{array}\right]_{N_{2} \times N_{2}}, \quad \mathbf{N}_{2 y}=\left[\begin{array}{ccc}
n_{y}\left(\mathbf{x}_{1}^{(2)}\right) & & 0 \\
& \ddots & \\
0 & & n_{y}\left(\mathbf{x}_{N_{2}}^{(2)}\right)
\end{array}\right]_{N_{2} \times N_{2}},
$$

$\mathbf{x}_{i}^{(2)} \in \Gamma_{2}, i=1 \sim N_{2}$

At the interface between the half-space soil and water layer $\left(\Gamma_{4}\right)$, the following continuity conditions for displacements and tractions hold (Fig. 1):

$\mathbf{U}_{4 z}^{(s)}=\mathbf{Q}_{n 4}, \mathbf{T}_{4 x}^{(s)}=\mathbf{T}_{4 y}^{(s)}=\mathbf{0}, \mathbf{T}_{4 z}^{(s)}=\mathbf{P}_{4}$

\subsection{Derivation of the Coupled BEM Model for the Pile- Soil-water System}

By using equations (12) and (19), the following equation for the pile is obtained:

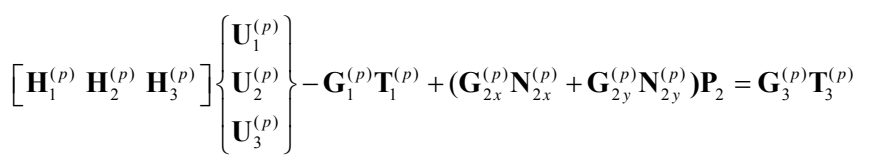

in which $\mathbf{G}_{2 x}^{(p)}$ and $\mathbf{G}_{2 y}^{(p)}$ are the sub-matrices of $\mathbf{G}_{2}^{(p)}$, corresponding to $\mathbf{T}_{2 x}^{(p)}$ and $\mathbf{T}_{2 y}^{(p)}$ in equation (14), respectively; as noted above, $\mathbf{T}_{3}^{(p)}$ at the right-hand side of equation (22) is assumed to be given by the boundary conditions. By using equation (15) as well as continuity conditions (18) and (21), the following equation for the half-space soil is obtained:

$$
\left[\begin{array}{ll}
\mathbf{H}_{1}^{(s)} & \mathbf{H}_{4}^{(s)}
\end{array}\right]\left\{\begin{array}{l}
\mathbf{U}_{1}^{(p)} \\
\mathbf{U}_{4}^{(s)}
\end{array}\right\}+\mathbf{G}_{1}^{(s)} \mathbf{T}_{1}^{(p)}-\mathbf{G}_{4 \mathrm{z}}^{(s)} \mathbf{P}_{4}=\mathbf{0}
$$

in which $\mathbf{G}_{4 z}^{(s)}$ is the sub-matrix of $\mathbf{G}_{4}^{(s)}$, corresponding to the traction vector $\mathbf{T}_{4 z}^{(s)}$ in equation (16). By using equation (17) as well as continuity conditions (19) and (21), the following equation is derived for the water layer:

$\mathbf{G}_{2}^{(w)}\left[\mathbf{N}_{2 x}^{(p)} \mathbf{N}_{2 y}^{(p)}\right]\left\{\begin{array}{l}\mathbf{U}_{2 x}^{(p)} \\ \mathbf{U}_{2 y}^{(P)}\end{array}\right\}+\mathbf{G}_{4}^{(w)} \mathbf{U}_{z 4}^{(s)}+\mathbf{G}_{5}^{(w)} \mathbf{Q}_{n 5}-\mathbf{H}_{2}^{(w)} \mathbf{P}_{2}-\mathbf{H}_{4}^{(w)} \mathbf{P}_{4}=\mathbf{0}$
Note that in deriving equation(24), the aforementioned traction free boundary condition along the surface of the water layer is used.

Finally, combination of equations (22), (23) and (24) yields the following equation for all unknown boundary variables of the pile-soil-water system:

$\left[\begin{array}{lll}\mathbf{E}_{1} & \mathbf{E}_{2} & \mathbf{E}_{3}\end{array}\right]\left\{\begin{array}{c}\Delta_{1} \\ \Delta_{2} \\ \Delta_{3}\end{array}\right\}=\left\{\begin{array}{c}\mathbf{G}_{3}^{(p)} \mathbf{T}_{3}^{(p)} \\ \mathbf{0} \\ \mathbf{0}\end{array}\right\}$

in which

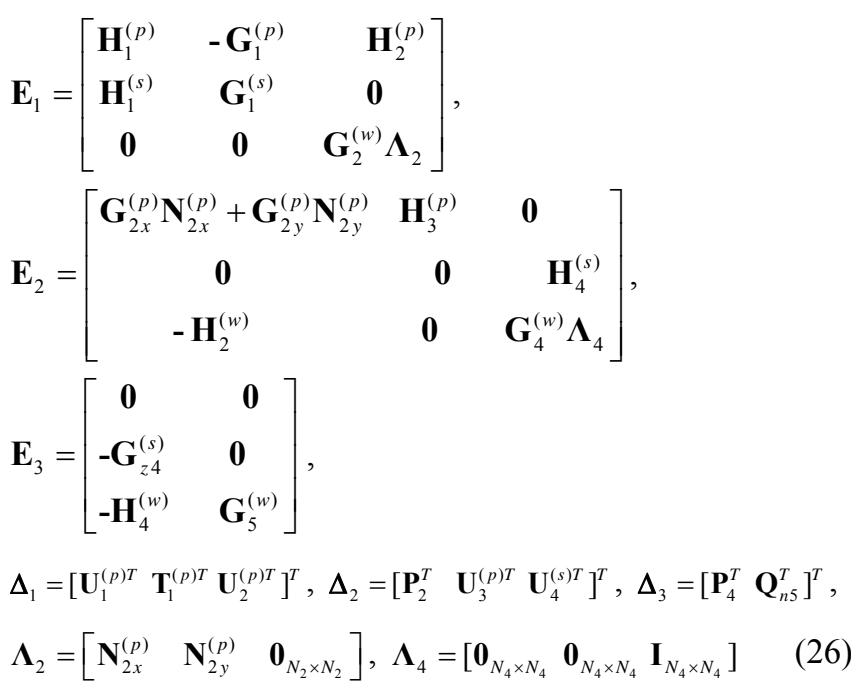

Solution of equation (25) gives all unknown variables along the boundaries of the pile-soil-water system. Once the boundary variables are determined, the variables at the internal points of the pile, half-space soil and water layer can be obtained using the corresponding discrete boundary integral equations for internal points.

\section{NUMERICAL METHOD AND NUMERICAL RE- SULTS FOR THE DYNAMIC RESPONSE OF THE PILE}

The coupled BEM model for the pile-soil-water system has been developed in the above section. In this section, numerical scheme for the coupled BEM model of the pile-soilwater system will be outlined briefly. Also, some numerical results obtained by the proposed model will be presented.

\subsection{Numerical Scheme for the BEM Model of the Pile- Soil-Water System}

The pile in this study is assumed to have a circular cross section (Fig. 1). The boundaries of the pile, half-space soil and water layer are discretized by the same 2-D eight-node isoparametric boundary elements [24], respectively. The bottom and top of the pile are discretized by twelve eightnode isoparametric elements, respectively, and the discretization scheme is showed in Fig. (2a). The side of the pile is divided into several segments evenly, and each segment of the pile is discretized by eight eight-node isoparametric elements evenly. The discretization of the interface between the half-space soil and water layer as well as the surface of the 
water layer is accomplished by truncating the corresponding infinite boundaries with several horizontal and vertical boundary element layers as schematically shown in Fig. (2b). Note that in order to discretize the above infinite boundaries economically, the sizes of the elements in different boundary element layers may increase gradually with increasing distance from the pile (Fig. (2b)).

\subsection{Numerical Results and Corresponding Analysis}

In this section, based on the proposed BEM model for the pile-soil-water system, five numerical examples will be presented. In the first example, the pile-soil-water system in this study is reduced to the common pile and half-space soil system by letting the thickness of the water layer approach zero. The solution for this special case of our model is compared with existing results for the pile half-space soil problem. In the second example, the influence of the pile-soil density ratio on the horizontal displacement and bending moment of the pile will be examined. In the third and fourth examples, the influences of the pile-soil modulus and density ratios on the compliances of the pile will be studied. In the fifth example, the influence of the water layer on the compliances of the pile will be examined. Note that in calculations, the typical values for the material and geometric parameters for the pile, half-space soil and water layer are given in Table $\mathbf{1}$.

The pile top displacements and the forces applied at the top of the pile are related to each other by the compliances of the pile, which can be represented by the following equation:

$$
\left\{\begin{array}{l}
\Delta_{V_{0}} \\
\Delta_{H_{0}} \\
\theta_{0} \\
\phi_{0}
\end{array}\right\}=\left[\begin{array}{cccc}
C_{V V} & 0 & 0 & 0 \\
0 & C_{H H} & C_{H M} & 0 \\
0 & C_{M H} & C_{M M} & 0 \\
0 & 0 & 0 & C_{T T}
\end{array}\right]\left\{\begin{array}{c}
F_{V_{0}} \\
F_{H_{0}} \\
M_{0} \\
T_{0}
\end{array}\right\}
$$

where $\Delta_{V_{0}}, \Delta_{H_{0}}, \theta_{0}$ and $\phi_{0}$ are the vertical displacement, horizontal displacement, rotation angle and twist angle at the top of the pile; $F_{V_{0}}, F_{H_{0}}, M_{0}$ and $T_{0}$ are the vertical force,

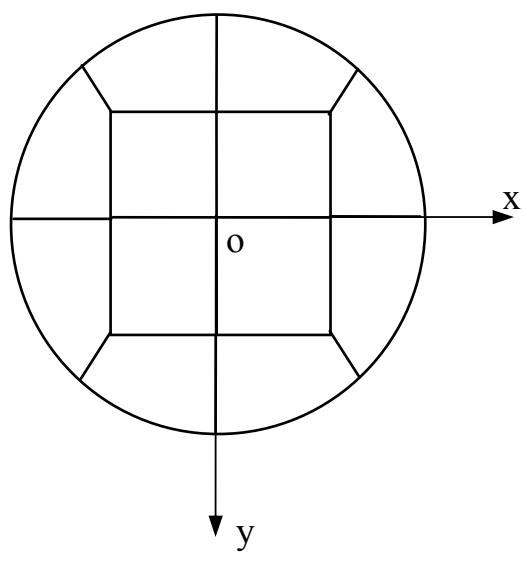

(a)
Table 1. Typical Values for the Parameters of the Pile, Halfspace Soil and Water Layer

\begin{tabular}{|c|c|}
\hline Parameters & Values \\
\hline \hline The shear modulus of the soil $\left(\mu_{s}\right)$ & $2.0 \times 10^{7} \mathrm{~Pa}$ \\
\hline The Poisson's ratio of the soil $\left(v_{s}\right)$ & 0.3 \\
\hline Density of the soil $\left(\rho_{s}\right)$ & $2.5 \times 10^{3} \mathrm{~kg} / \mathrm{m}^{3}$ \\
\hline The bulk modulus of the water $\left(K_{w}\right)$ & $2.0 \times 10^{9} \mathrm{~Pa}$ \\
\hline The density of the water $\left(\rho_{w}\right)$ & $1.0 \times 10^{3} \mathrm{~kg} / \mathrm{m}^{3}$ \\
\hline The shear modulus of the pile $\left(\mu_{p}\right)$ & $2.0 \times 10^{10} \mathrm{~Pa}$ \\
\hline The Poisson's ratio of the pile $\left(v_{p}\right)$ & 0.3 \\
\hline The density of the pile $\left(\rho_{p}\right)$ & $2.5 \times 10^{3} \mathrm{~kg} / \mathrm{m}^{3}$ \\
\hline The diameter of the pile $\left(d_{p}\right)$ & $2 \mathrm{~m}$ \\
\hline $\begin{array}{c}\text { The length of the pile immersed in the water layer } \\
\left(L_{p 1}\right)\end{array}$ & $10 \mathrm{~m}$ \\
\hline $\begin{array}{c}\text { The length of the pile embedded in the half-space } \\
\text { soil }\left(L_{p 2}\right)\end{array}$ & $20 \mathrm{~m}$ \\
\hline
\end{tabular}

horizontal force, moment and torque applied at the top of the pile; $C_{V V}, C_{H H}, C_{H M}, C_{M H}, C_{M M}$ and $C_{T T}$ are the compliances of the pile. Note that due to the reciprocity theorem in elastodynamics [25], compliances $C_{H M}$ and $C_{M H}$ in equation (27) are equal. As the pile in equation (27) is considered as a one-dimensional structure, the vertical displacement $\Delta_{V_{0}}$ and twist angle $\phi_{0}$ thus only depend on the vertical force and torque, respectively. Since the pile in this study is treated as a three-dimensional structure, the pile top displacements $\Delta_{V_{0}}, \Delta_{H_{0}}, \theta_{0}$ and $\phi_{0}$ in equation (27) should be considered as the one-dimensional equivalent displacements to the corresponding three-dimensional counterparts, which can be

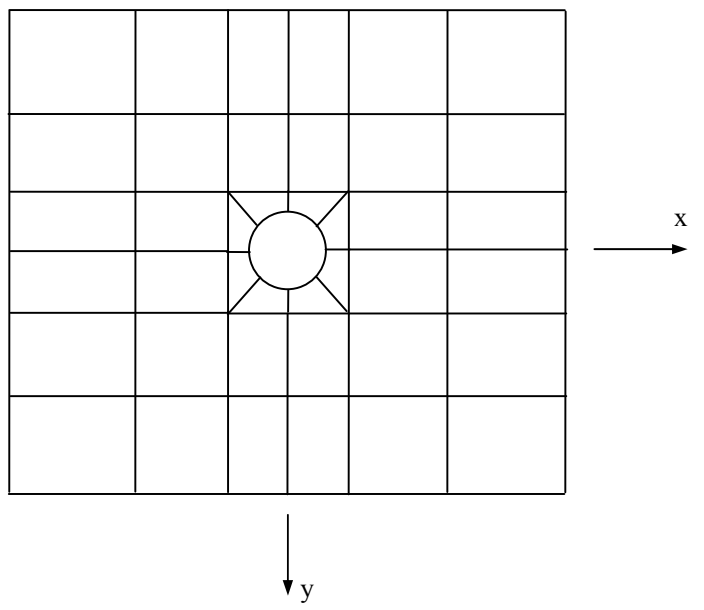

(b)

Fig. (2). The BEM discretization scheme for the top and bottom of the pile as well as the interface between the half-space soil and water layer and the surface of the water layer: (a) the discretization for the top and bottom of the pile; (b) the discretization for the interface between the half-space soil and water layer as well as the surface of the water layer. 
determined by using the corresponding three-dimensional displacements of the boundary elements at the pile top. Likewise, the forces $F_{V_{0}}, F_{H_{0}}, M_{0}$ and $T_{0}$ should be considered as the resultant forces of the tractions applied at the boundary elements of the pile top.

\subsubsection{Comparison of Present Results with Existing Results}

As noted above, when the thickness of the water layer tends to zero, the pile-soil-water system in this study is reduced to the common pile half-space soil system. The compliance $C_{V V}$ of the pile is calculated for this special case and compared with existing results [14]. The compliance function $C_{V V}$ is normalized with respect to the corresponding static compliance $C_{V V 0}$ for the same pile, and the results are plotted versus the dimensionless frequency $a_{0}$ (Fig. 3). The dimensionless frequency $a_{0}$ is defined as follows

$a_{0}=\frac{\omega d_{p}}{v_{s}}$

where $d_{p}$ is the diameter of the pile and $v_{s}$ the shear wave velocity of the half-space soil. In the numerical simulation, the pile is divided evenly into twenty segments. Sixteen horizontal and sixteen vertical boundary element layers are used in the discretization of the interface between the halfspace soil and water layer as well as the surface of the water layer (Fig. 2). Fig. (3) illustrates that there is very good agreement between the results of this paper and those presented by Mamoon et al. [14], validating the proposed model of this paper.

\subsubsection{The Influence of the Pile-Soil Density Ratio on the Dynamic Response of the Pile}

In this section, the influence of the pile-soil density ratio on the dynamic response of the pile will be examined. Except the density of the pile, other parameters for the pile, half-space soil and water layer take the typical values as given in Table 1 . The density of the pile $\left(\rho_{p}\right)$ takes the values to make $\rho_{p} / \rho_{s}=1.0,1.4,1.7$, respectively, in which

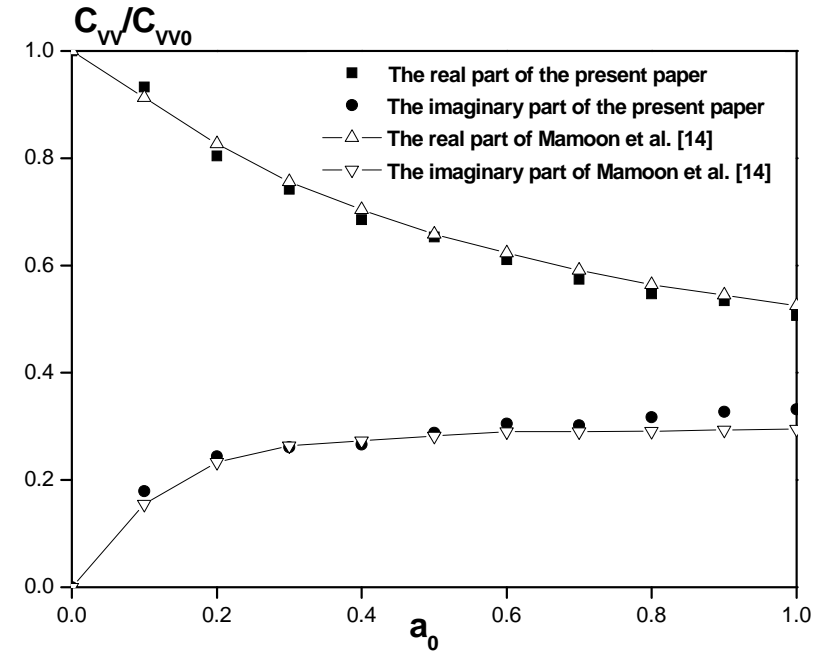

Fig. (3). Comparison of present results for the compliance $C_{V V} / C_{V V 0}$ of a pile embedded in a half-space soil with the following parameters: $L / d=20, \quad E_{p} / E_{s}=100, \quad \rho_{s} / \rho_{p}=0.7$ with the results due to Mamoon et al. [14].

the subscripts $p$ and $s$ denote the pile and half-space soil, respectively. A unit horizontal load is applied at the top of the pile, and the frequency is equal to $5 \mathrm{~Hz}$. In the numerical simulation, the pile embedded in the soil is divided evenly into twenty five segments and the part immersed in the water layer is divided evenly into thirteen segments. Sixteen horizontal and sixteen vertical boundary element layers are used in the discretization of the interface between the half-space soil and water layer as well as the surface of the water layer (Fig. 2).

Fig. (4) plots the amplitudes of the horizontal displacement and bending moment of the pile for the three different density ratios $\left(\rho_{p} / \rho_{s}\right)$. Fig. (4a) shows that when $z /\left(L_{p 1}+L_{p 2}\right)<0.55$, the horizontal displacement of the pile has a steady decrease with increasing depth. However, when

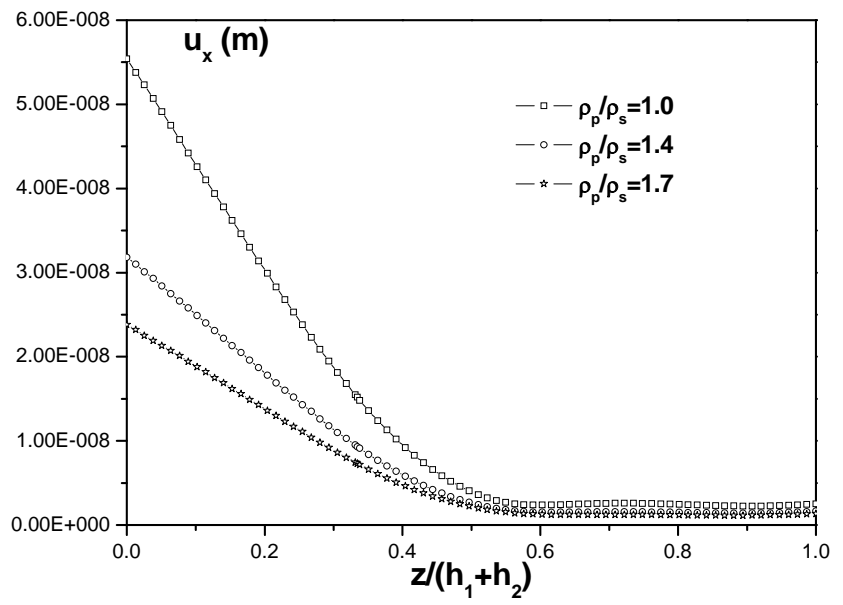

(a)

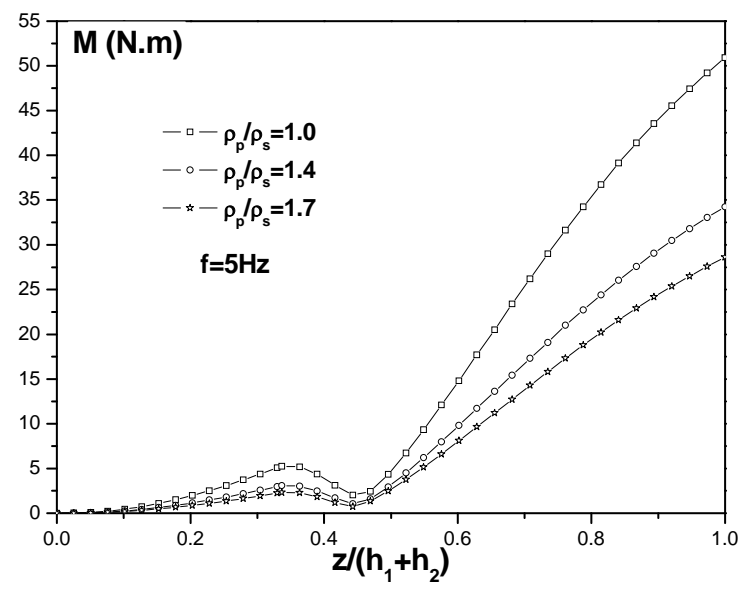

(b)

Fig. (4). The influence of the pile-soil density ratio on the dynamic response of the pile when the top of the pile is subjected to a horizontal load with frequency $f=5 \mathrm{~Hz}$ : (a) the horizontal displacement of the pile; (b) the bending moment of the pile. 
$z /\left(L_{p 1}+L_{p 2}\right)>0.55$, the horizontal displacement of the pile almost keeps unchanged. Fig. (4a) also illustrates that when $z /\left(L_{p 1}+L_{p 2}\right)<0.55$, the horizontal displacement of the pile decreases considerably with increasing density ratio $\left(\rho_{p} / \rho_{s}\right)$. Moreover, it follows from Fig. (4b) that when $z /\left(L_{p 1}+L_{p 2}\right)<0.45$, the bending moment of the pile is small. Nevertheless, when $z /\left(L_{p 1}+L_{p 2}\right)>0.45$, the bending moment of the pile almost increases linearly with increasing depth. Besides, when $z /\left(L_{p 1}+L_{p 2}\right)>0.45$, the bending moment of the pile decreases pronouncedly with the increase of the density ratio $\left(\rho_{p} / \rho_{s}\right)$.

\subsubsection{The Influence of the Pile-Soil Modulus Ratio on the Compliances of the Pile}

In this section, based on the proposed BEM model for the pile-soil-water system, the influence of the pile-soil modulus ratio on the compliances of the pile is investigated. The compliances of the pile are defined in equation (27). Except the modulus of the pile $\left(E_{p}\right)$, all material and geometric parameters for the pile, half-space soil and water layer assume the typical values as given by Table $\mathbf{1}$. The modulus of the pile takes the values to make $E_{p} / E_{s}=100,500,1000$, respectively. In the numerical simulation, the boundary discretization schemes for the pile, half-space soil and water layer are the same as those in Section 5.2.2.

Fig. (5) illustrates the compliances of the pile versus the dimensionless frequency. Also, as noted above, as the compliances $C_{H M}$ and $C_{M H}$ are equal to each other, the compliance $C_{M H}$ is thus omitted in Fig. (5). Fig. (5a) shows that with increasing modulus ratio $E_{p} / E_{s}$, the real part of the compliance $C_{V V}$ decreases considerably, while the magnitude of the imaginary part decreases slightly. Figs (5b-d) shows that the real and imaginary parts of compliances increases slightly in the frequency range $0 \leq a_{0} \leq 0.2$. In the frequency range $0.2 \leq a_{0} \leq 0.7$, the displayed compliances of the pile oscillate and a resonance peak occurs for each modulus ratio. Furthermore, as expected, with increasing modulus ratio, the resonant frequency increases. In the frequency range $0.7 \leq a_{0} \leq 1.0$, the amplitudes of compliances decrease with increasing frequency. It follows from Fig. (5e) that both the real and imaginary parts of the compliance $C_{T T}$ increase slightly with the increase of frequency and the compliance increases more pronouncedly when the modulus ratio takes smaller value.

\subsubsection{The Influence of the Pile-Soil Density Ratio on the Compliances of the Pile}

In this section, the influence of the pile-soil density ratio on the compliances of the pile will be investigated. Except the density of the pile, all material and geometric parameters for the pile, half-space soil and water layer take the typical values as given by Table 1 . The density of the pile $\left(\rho_{p}\right)$ takes the values making $\rho_{p} / \rho_{s}=1.0,1.4,1.7$, respectively. In the numerical simulation, the boundary discretization schemes for the pile, half-space soil and water layer are the same as those in Section 5.2.2.

Fig. (6a) illustrates that the real part of the compliance $C_{V V}$ decreases and the imaginary part increases with the increase of frequency. At the low frequency range, the differences between the compliances for different density ratios are minor. However, with increasing frequency, the differences between the compliances for different density ratios become pronounced. Also, it follows from Figs (6b-d), in the frequency range $0 \leq a_{0} \leq 0.3$, both the real and imaginary parts of the displayed compliances show a slight increase with increasing frequency. In the frequency range $0.3 \leq a_{0} \leq 0.7$, the presented compliances oscillate and as with Section 5.2.3, a resonance peak arises for each density ratio. In contrast to Section 5.2.3, with increasing density ratio, the resonant frequency decreases. Fig. (6e) shows that both the real and imaginary parts of the torsional compliance $C_{T T}$ increase with the increase of frequency. Also, it follows that the density ratio $\rho_{p} / \rho_{s}$ does not have an obvious influence on the torsional compliance of the pile.

\subsubsection{The Influence of the Water Layer on the Compli- ances of the Pile}

In this section, the influence of the water layer on the compliances of the pile will be examined. To investigate the influence of the water layer, three cases for the pile are considered in this section, namely, the case for the pile embedded in the half-space soil covered by the water layer (the soil-water case), the case wherein the water layer is replaced by the soil (the soil case), and the case in which the water layer is absent (the absent case), i.e., the water layer is replaced by the air. Note that the second case corresponds to the circumstance in which the pile is embedded in the halfspace soil, while the third case corresponds to the situation wherein the pile is extended above the surface of the halfspace soil. In all three cases, the total length of the pile is equal to $20 \mathrm{~m}$. In the first case, the lengths of the segments of the pile embedded in the half-space soil and immersed in the water layer are both equal to $10 \mathrm{~m}$, respectively. The segments of the pile embedded in the soil and immersed in the water layer are both divided evenly into 13 segments. In the second case, the 20-meter long pile embedded in the halfspace soil is divided evenly into 26 segments. In the third case, the 10-meter long segment of the pile embedded in the soil is divided evenly into 13 segments, and the 10 -meter long extended segment of the pile in the air is also divided evenly into 13 segments. Other parameters for the pile, halfspace soil and water layer take the typical values as given by Table 1. The discretization schemes for the boundary of the pile, half-space soil and water layer are the same as those in Section 5.2.2. 


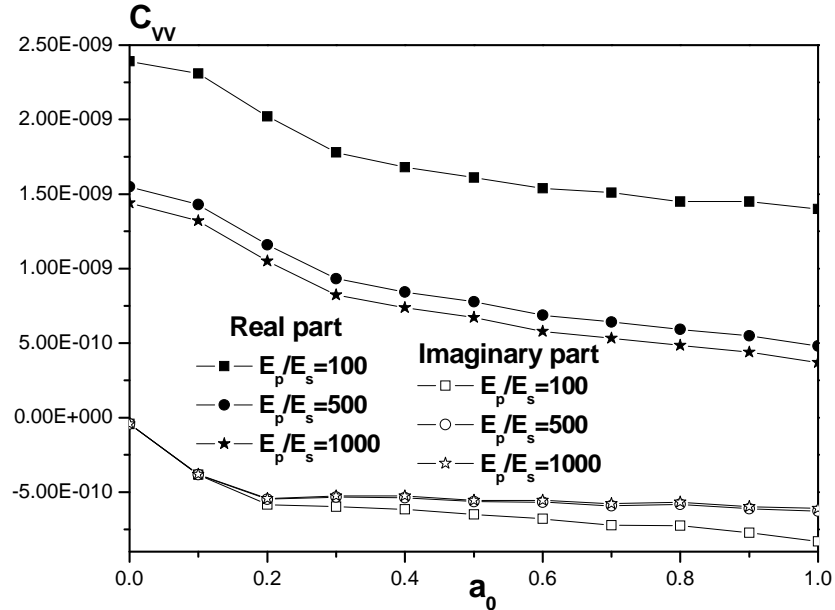

(a)

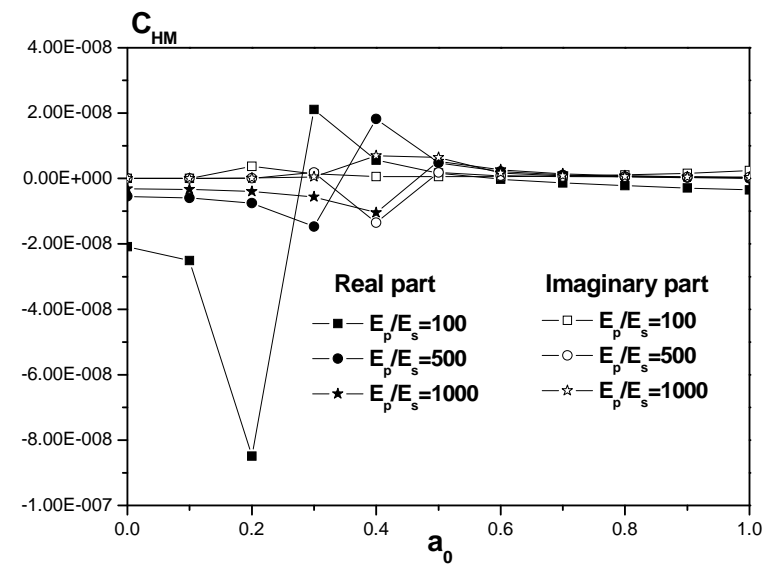

(c)

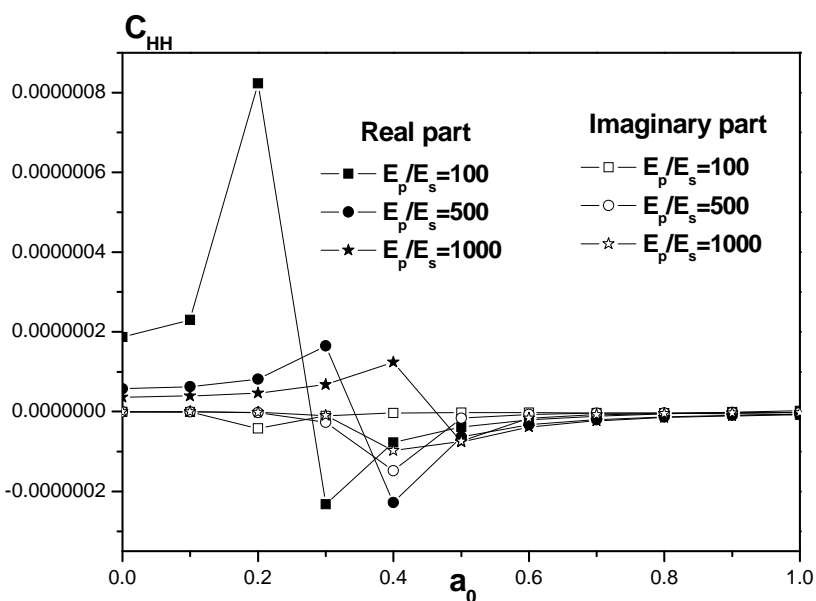

(b)

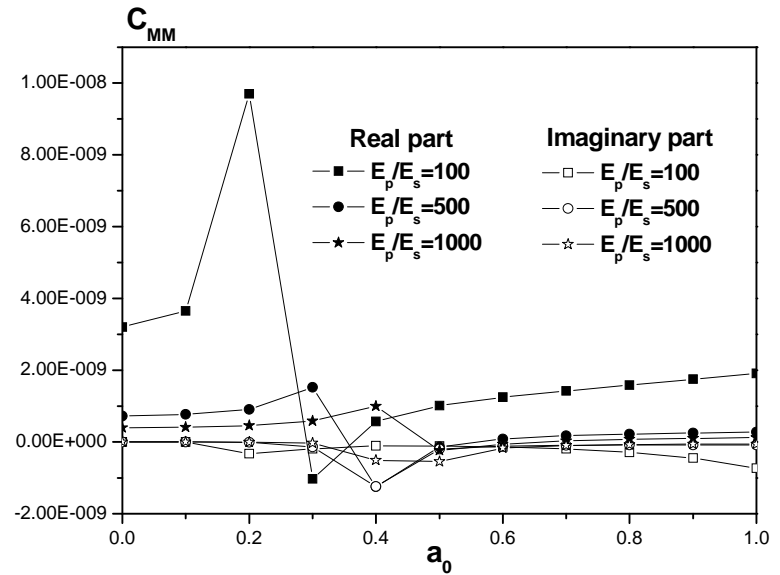

(d)

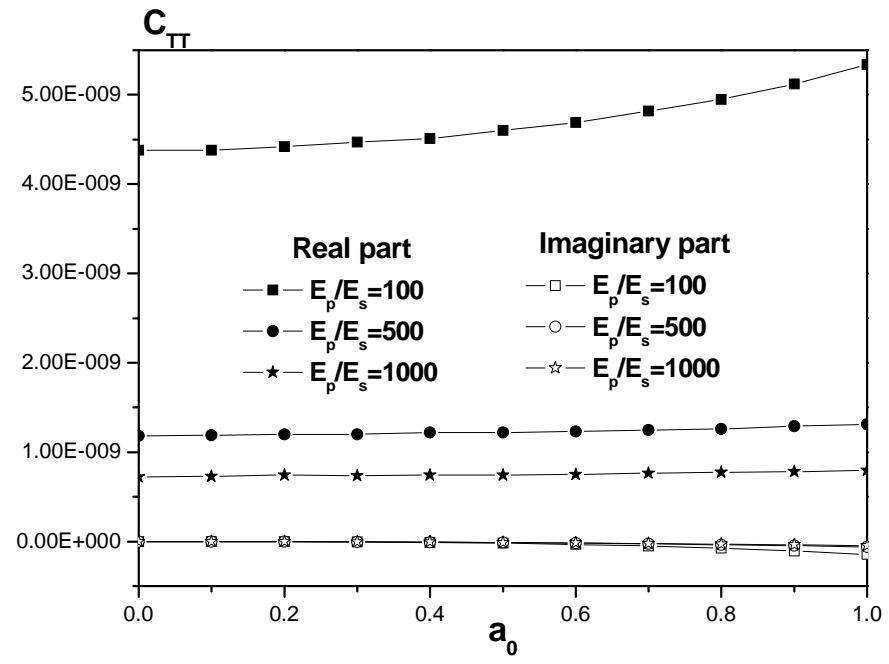

(e)

Fig. (5). The compliances of the pile embedded in a half-space soil covered by a water layer when $E_{p} / E_{s}=100,500$ and 1000 , respectively and other parameters for the pile-soil-water system take the typical values as given by Table 1: (a) the compliances $C_{V V} ;(\mathbf{b})$ the compliances $C_{H H} ;(\mathbf{c})$ the compliances $C_{H M} ;(\mathbf{d})$ the compliances $C_{M M} ;(\mathbf{e})$ the compliances $C_{T T}$. 


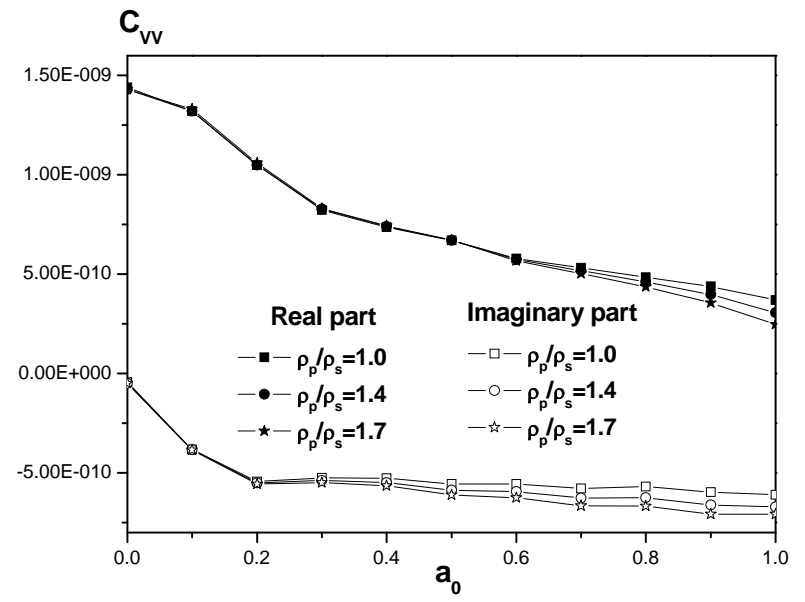

(a)

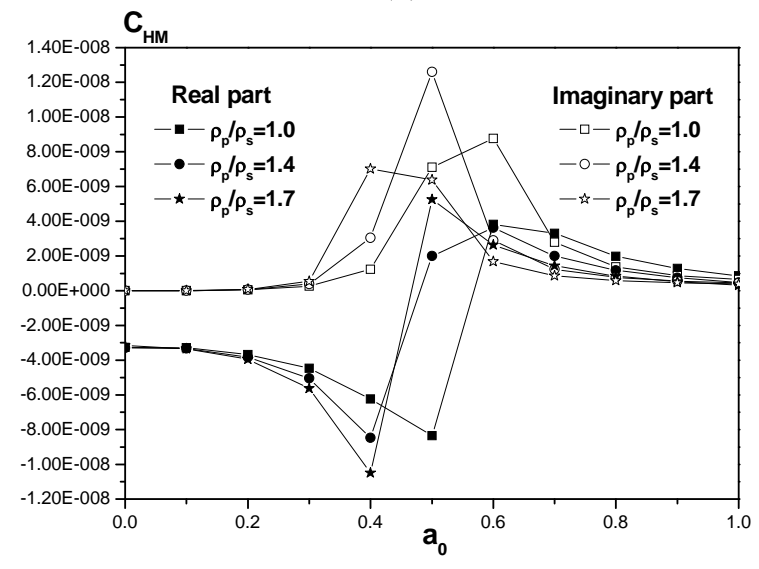

(F)

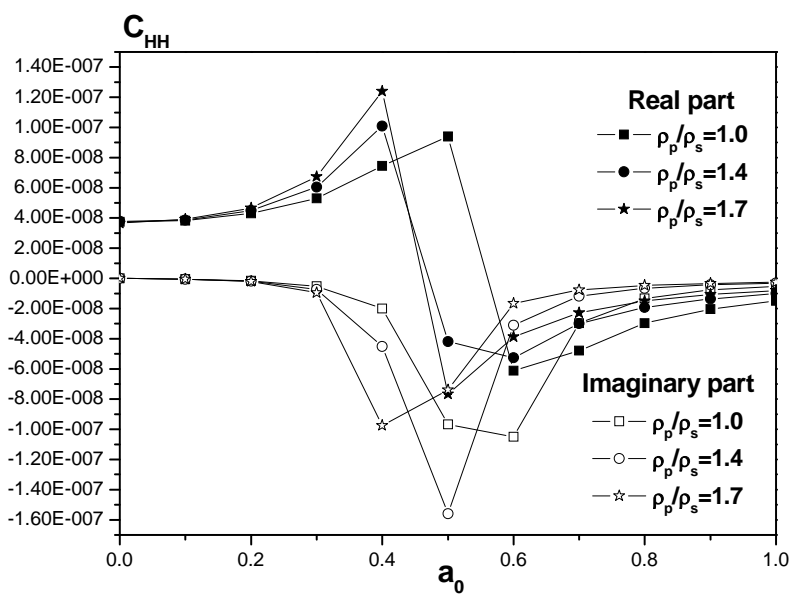

(E)

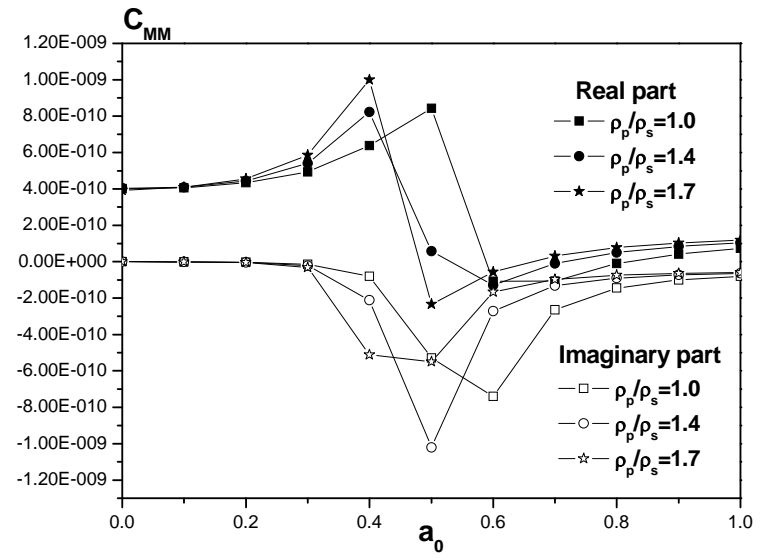

(G)

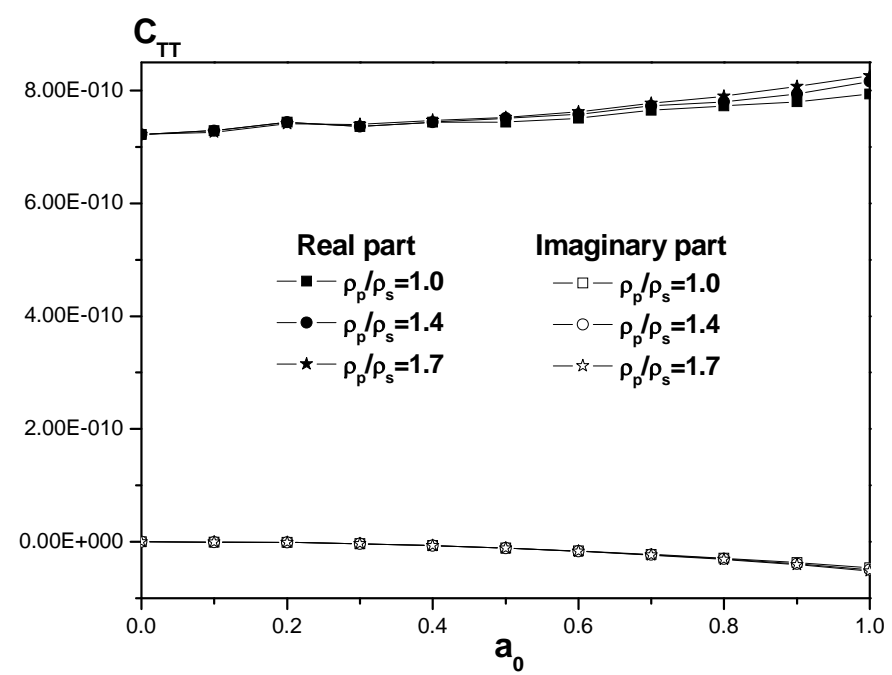

(H)

Fig. (6). The compliances of the pile embedded in a half-space soil covered by a water layer when $\rho_{p} / \rho_{s}=1.0,1.4$ and 1.7 , respectively and other parameters of the pile-soil-water system take the typical values as given by Table $1:$ (a) the compliances $C_{V V} ;(\mathbf{b})$ the compliances $C_{H H} ;(\mathbf{c})$ the compliances $C_{H M} ;(\mathbf{d})$ the compliances $C_{M M} ;(\mathbf{e})$ the compliances $C_{T T}$. 


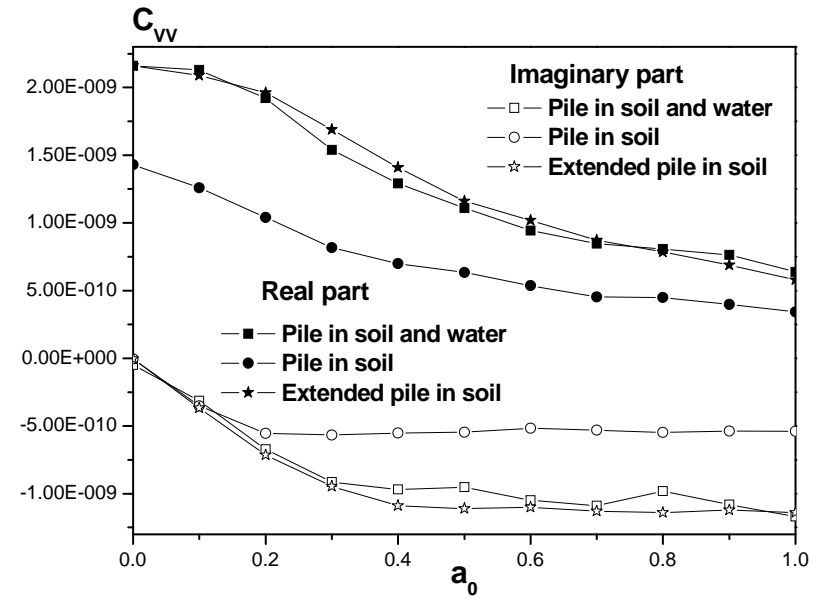

(D)

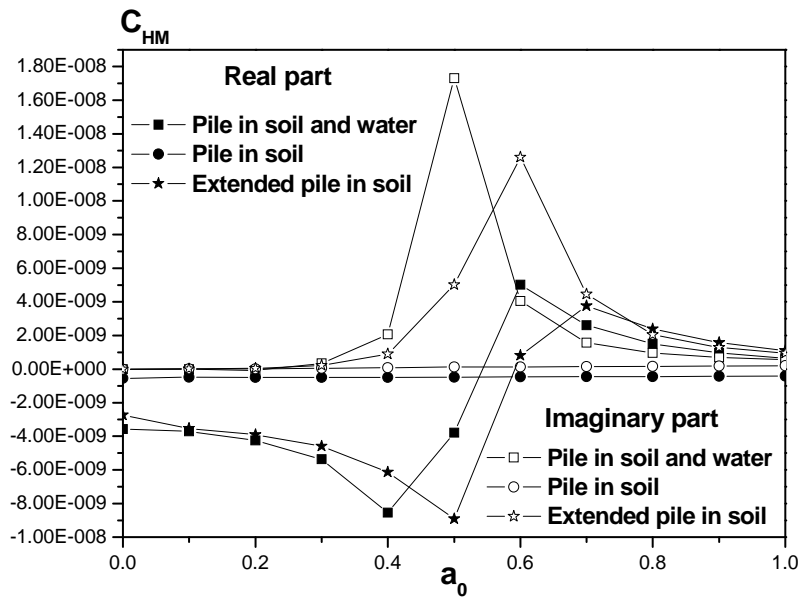

(F)

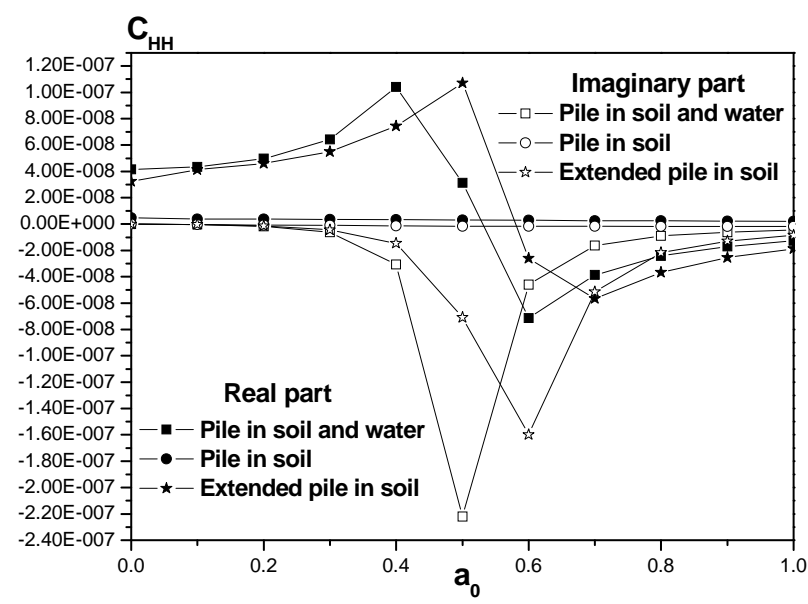

(E)

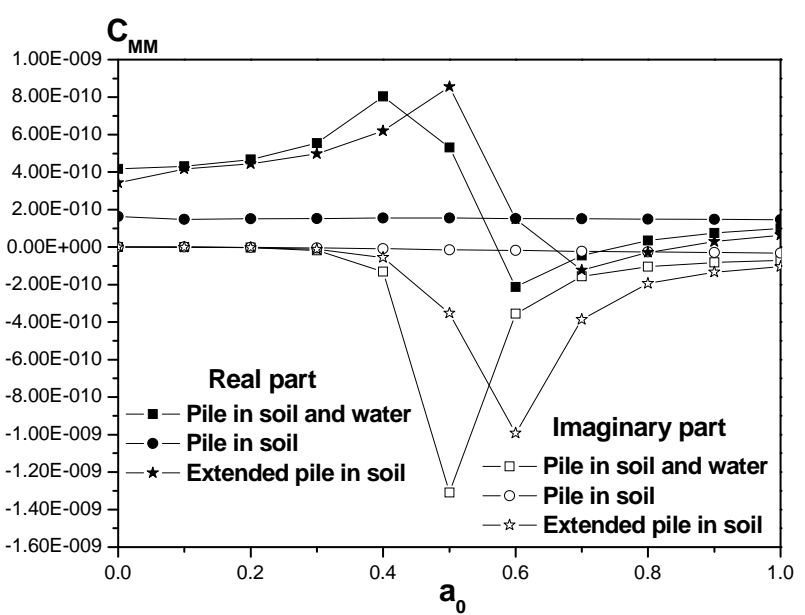

(G)

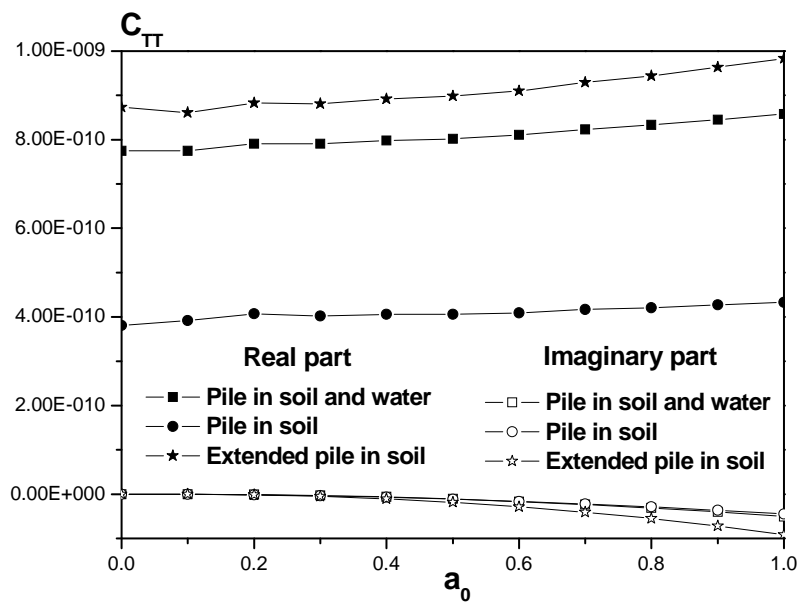

(H)

Fig. (7). The compliances of the pile for the three cases, namely, the case for the pile embedded in the half-space soil covered by the water layer, the case wherein the water layer is replaced by the soil, and the case in which the water layer is absent: (a) the compliances $C_{V V} ;(\mathbf{b})$ the compliances $C_{H H} ;(\mathbf{c})$ the compliances $C_{H M} ;(\mathbf{d})$ the compliances $C_{M M} ;(\mathbf{e})$ the compliances $C_{T T}$. 
Fig. (7a) illustrates that the real parts of the compliance $C_{V V}$ decrease and the magnitudes of the imaginary parts increase with the increase of frequency. The difference of the compliance $C_{V V}$ between the soil-water case and the absent case is minor. However, both the real and imaginary parts of the compliance $C_{V V}$ for the soil case are smaller than those of the other two cases, suggesting that the pile in the soil case is stiffer than those in the other two cases and also in this case, less energy is radiated to the half-space soil due to the larger length of the pile segment embedded in the soil. Figs (7b-d) show that the magnitudes of the compliances $C_{H H}, C_{H M}$ and $C_{M M}$ of the soil case is much smaller than those of the other two cases, implying again that the pile in the soil case is stiffer and less damping than those in the other two cases. Figs (7b-d) also show that the resonance frequencies of the soil-water case are smaller that those of the absent case, meaning that the water layer in this situation mainly functions as a mass. Fig. (7e) shows that compared with the real parts, the imaginary parts of the torsional compliance $C_{T T}$ of the three cases are smaller, and the differences between the imaginary parts of the compliance $C_{T T}$ of the three cases are little. However, the differences between the real parts of the compliance $C_{T T}$ of the three cases are significant. As expected, the real part of the compliance of the absent case is the largest, while that of the soil case is the smallest.

\section{CONCLUSIONS}

A frequency domain coupled BEM model for a pile embedded in a half-space soil covered by a water layer has been proposed in this study. Obviously, the model developed in this study can be used in the analysis of the dynamic response of bridges, docks and offshore platforms to various dynamic loads when the pile-soil-water coupling is considered. Based on the proposed model, some numerical results are presented. By the researches conducted in this paper, the following conclusions are drawn:

1). Comparison of results of this paper with existing results for a single pile embedded in a half-space soil validates the coupled BEM model for the pile-soilwater system proposed in this study.

2). When the pile is subjected to a horizontal load, the horizontal displacement of the segment of the pile immersed in the water layer are much larger than that of the segment embedded in the half-space soil. In contrast to the horizontal displacement, the bending moment of the segment of the pile immersed in the water layer is much smaller than that of the segment embedded in the half-space soil.

3). In the calculated frequency range, when subjected to axial load and torque, the pile does not exhibit obvious resonance phenomena. However, when subjected to horizontal load and moment, the pile shows obvious resonance phenomena, with resonant frequency increasing with increasing pile-soil modulus ratio and decreasing with increasing pile-soil density ratio.
4). The comparison between the compliances for the three cases, namely, the soil-water case, soil case and absent case show that the stiffness of the pile of the soil case is the largest among the three, and the damping of the pile of the soil case is the smallest. Also, numerical results of the compliances for the piles of the soil-water and absent cases show that the mass effect of the water layer is significant and dominant, while the stiffness effect of the water layer is almost negligible.

5). Although only the frequency domain response of the pile to the loads acting on the pile top is investigated in this study, the response of the pile to other kinds of loads, such as, seismic waves, current loads and water waves etc, can also be analyzed by the proposed model. By means of the Fourier transform method, the proposed model can also be used to investigate the time domain response of the pile. Moreover, by the procedure proposed in this study, the problem for pile groups embedded in a half-space soil overlain by a water layer can also be solved in a straightforward way.

\section{CONFLICT OF INTEREST}

The authors confirm that this article content has no conflicts of interest.

\section{ACKNOWLEDGEMENTS}

This research is conducted in the framework of a project supported by the National Natural Science Foundation of China with grant number 51078171. Also, the constructive comments from two referees are highly acknowledged by the authors.

\section{APPENDIX}

\section{The Frequency Domain Green's Functions for a Three- Dimensional Elastic Medium}

The frequency domain Green's functions $U_{i j}$ and $T_{i j}$ for an elastic medium have the following form [21]

$$
\begin{aligned}
& U_{i j}^{(G)}=\frac{1}{4 \pi \mu}\left(\psi \delta_{i j}-\chi r_{, i} r_{, j}\right), \\
& T_{i j}^{(G)}=\frac{1}{4 \pi}\left[A\left(\delta_{i j} \frac{\partial r}{\partial \mathbf{n}}+r_{, j} n_{i}\right)+B\left(n_{j} r_{, i}-2 r_{, i} r_{, j} \frac{\partial r}{\partial \mathbf{n}}\right)+C r_{, j} r_{, i} \frac{\partial r}{\partial \mathbf{n}}+D r_{, i} n_{j}\right]
\end{aligned}
$$

and

$$
\begin{aligned}
& \begin{array}{l}
\psi=\left(-\frac{C_{2}^{2}}{\omega^{2} r^{2}}-\mathrm{i} \frac{C_{2}}{\omega r}+1\right) \frac{e^{-\mathrm{i} \omega r / C_{2}}}{r}+\frac{C_{2}^{2}}{C_{1}^{2}}\left(\frac{C_{1}^{2}}{\omega^{2} r^{2}}+\mathrm{i} \frac{C_{1}}{\omega r}\right) \frac{e^{-\mathrm{i} \omega r / C_{1}}}{r}, \\
\chi=\left(-\frac{3 C_{2}^{2}}{\omega^{2} r^{2}}-\mathrm{i} \frac{3 C_{2}}{\omega r}+1\right) \frac{e^{-i \omega r / C_{2}}}{r}+\frac{C_{2}^{2}}{C_{1}^{2}}\left(\frac{3 C_{1}^{2}}{\omega^{2} r^{2}}+\mathrm{i} \frac{3 C_{1}}{\omega r}-1\right) \frac{e^{-\mathrm{i} \omega r / C_{1}}}{r} \\
\text { where } r=\sqrt{x_{i} X_{i}}, \quad A=d \psi / d r-\chi / r, \quad B=-2 \chi / r, \\
C=-2 d \chi / d r, D=\left(C_{1}^{2} / C_{2}^{2}-2\right)(d \psi / d r-d \chi / d r-2 \chi / r)
\end{array}
\end{aligned}
$$


, and $C_{1}, C_{2}$ are the compressive and shear wave velocities of the elastic medium, respectively.

\section{REFERENCES}

[1] N. Makris, "Soil-pile interaction during the passage of rayleigh waves: An analytical solution", Earthquake. Eng. Struct., vol. 23, pp. 153-167, 1994.

[2] N. Makris and D. Badoni, "Seismic response of pile groups under oblique-shear and Rayleigh waves", Earthquake. Eng. Struct., vol. 24, pp. 517-532, 1995.

[3] R.L. Kuhlemeyer, "Vertical vibration of piles", J. Geotech. Geoenviron., vol. 105, pp. 273-287, 1979.

[4] R.L. Kuhlemeyer, "Static and dynamic laterally loaded floating piles", J. Geotech. Geoenviron., vol. 105, pp. 289-304, 1979.

[5] G.X. Wu and W.D.L. Finn, "Dynamic elastic analysis of pile foundations using finite element method in the frequency domain", Can. Geotech. J., vol. 34, pp. 34-43, 1997.

[6] X. Zeng and R.K.N.D. Rajapakse, "Dynamic axial load transfer from elastic bar to poroelastic medium", J. Eng. Mech., vol. 125, pp. 1048-1055, 1999.

[7] R.K.N.D. Rajapakse "A note on the elastodynamic load transfer problem”, Int. J. Solids. Struct., vol. 24, pp. 963-972, 1988.

[8] R.K.N.D. Rajapakse and A.H. Shah, "On the longitudinal harmonic motion of an elastic bar embedded in an elastic half-space", Int. J. Solids. Struct., vol. 23, pp. 267-285, 1987.

[9] R.K.N.D. Rajapakse and A.H. Shah, "On the lateral harmonic motion of an elastic bar embedded in an elastic half-space", Int. J. Solids. Struct., vol. 23, pp. 287-303, 1987.

[10] Y. Cai, G. Chen, C. Xu and D. Wu, "Torsional response of pile embedded in a poroelastic medium", Soil. Dyn. Earthquake. Eng., vol. 26, pp. 1143-1148, 2006.

[11] B. Jin, D. Zhou and Z. Zhong, "Lateral dynamic compliance of pile embedded in poroelastic half space", Soil. Dyn. Earthq. Eng., vol. 21, pp. 519-525, 2001.
[12] J.F. Lu and D.S. Jeng, "Poro-elastic model for pile-porous medium interfaction due to seismic waves", Int. J. Numer. Anal. Methods. Geomech., vol. 32, pp. 1-41, 2008.

[13] R.Y.S. Pak and P.C. Jennings, "Elastodynamic response of pile under transverse excitations", J. Eng. Mech., vol. 113, pp. 11011116, 1987.

[14] S.M. Mamoon, A.M. Kaynia and P.K. Banerjee, "Frequency domain dynamic analysis of piles and pile groups", J. Eng. Mech., vol. 116, pp. 2237-2257, 1990.

[15] R. Sen, T.G. Davies and P.K. Banerjee, "Dynamic analysis of piles and pile groups embedded in homogeneous soils", Earthquake. Eng. Struct., vol. 13, pp. 53-65, 1985.

[16] H.C. Wang and P.K. Banerjee, "Generalized axisymmetric elastodynamic analysis by boundary element method", Int. J. Numer. Methods. Eng., vol. 30, pp. 115-131, 1990.

[17] O. Maeso, J.J. Aznárez and F. García, "Dynamic impedances of piles and groups of piles in saturated soils", Comput. Struct., vol. 83, pp. 769-782, 2005.

[18] A. Papoulis, The Fourier Integral and Its Application, McGrawHill: New York, 1962.

[19] J.D. Achenbach, Wave Propagation in Elastic Solids, NorthHolland: Holanda, 1973.

[20] G.D. Manolis and D.E. Beskos, Boundary Element Methods in Elastodynamics, Unwin Hyman: London, 1988.

[21] P.K. Banerjee, S. Ahmad and K. Chen, "Advanced application of BEM to wave barriers in multi-layered three-dimensional soil media", Earthquake. Eng. Struct., vol. 16, pp. 1041-1060, 1988.

[22] R.D. Ciskowski and C.A. Brebbia, Boundary Element Methods in Acoustics, Computational Mechanics Publications: Southampton, 1991.

[23] A.F. Seybert and T.W. Wu, "Modified Helmholtz integral equation for bodies sitting on an infinite plane", J. Acoust. Soc. Am., vol. 85, pp. 19-23, 1989.

[24] G. Beer, L. Smith and C. Duenser, The Boundary Element Method with Programming: For Engineers and Scientists, Springer: Berlin, 2008.

[25] A.C. Eringen and E.S. Suhubi, Elastodynamics, Volume II: Linear Theory, Academic Press: New York, 1975.

(C) Zhang and Lu; Licensee Bentham Open.

This is an open access article licensed under the terms of the Creative Commons Attribution Non-Commercial License (http://creativecommons.org/licenses/ by-nc/3.0/) which permits unrestricted, non-commercial use, distribution and reproduction in any medium, provided the work is properly cited. 